\title{
Minimizing Collateral Damage from COVD-19 Distancing Strategies: Protecting Patients who have Multi- Morbidity
}

\author{
Efrat Shadmi ${ }^{1}$, Chad Boult ${ }^{2 *}$ and Ran D Balicer ${ }^{3}$ \\ ${ }^{1}$ The Cheryl Spencer Department of Nursing, Faculty of Social Welfare and Health Sciences, University of Haifa, Mount Carmel, Israel \\ ${ }^{2}$ Board of Directors, International Foundation for Integrated Care, USA \\ ${ }^{3}$ Clalit Research Institute, Clalit Health Services, Shoham 2, Ramat Gan, Israel
}

The world is battling the novel coronavirus primarily with social distancing and hygienic strategies, a battle that will continue for many more months at a minimum. As in all wars, there will be some "collateral damage," but there are opportunities for healthcare systems to minimize such damage to many people.

Most epidemiologic models project that only a small fraction of the world's billion people who have "multi-morbidity" (i.e., three or more chronic health conditions) [1] will succumb to COVID-19-induced mortality. Many more multi-morbid people, most of whom are older, are vulnerable to incurring "collateral damage," i.e., deterioration and death as a result of "social-distancing" and other pandemic control strategies that are likely to continue until a vaccine becomes widely available (in mid-2021 at the earliest) [2].

These pandemic control strategies, while effective and essential for controlling the spread of viruses, often disrupt the management of multi-morbidity by:

- Decreasing access to primary health care (older patients' visits declined by $28 \%$ from early March to early May of 2020) [3], resulting in less in-person monitoring and managing of chronic conditions and medication side effects

- Decreasing access to medical equipment and community services, e.g., meals, aides and transportation to healthcare facilities, pharmacies and grocery stores

- Interrupting "coaching" programs that help chronically ill patients to adhere to their recommended diets, exercise regimens, medications and self-monitoring activities

- Decreasing the provision of essential emotional support, information and guidance for family caregivers

- Impeding the in-person expression of emotional support from family and friends

- Fragmenting all of these important processes, which are collectively much more effective when fully integrated.

Even in the best of times, providing and integrating these critical components of high-quality, comprehensive care for patients with multimorbidity is challenging. When access to such services becomes even less available, however, many multi-morbid patients will have difficulty surviving, especially those who depend on support for managing healthrelated behaviors, ongoing medical care, supportive equipment and community services [4]. The diminution of these essential components of care will lead to exacerbations of heart and kidney failure, COPD, dysrhythmias, diabetes, hypertension, malnutrition, depression and caregiver abuse/neglect - and increases in strokes, falls, myocardial infarctions, undetected/unmanaged cancers, medication adverse effects, substance abuse and suicide. Large increases in death rates from heart disease, diabetes, cerebrovascular disease and Alzheimer disease have already occurred in the U.S. [5].

Thus, containing the novel coronavirus by executing the currently available strategies is likely to lead inadvertently to significant increases in deterioration and death among the hundreds of millions of people with multi-morbidity who do not become infected with the virus unless specific counter-measures are implemented soon.

Two such counter-measures could be launched immediately. The first is the widespread implementation of electronic technologies, such as:

- Electronic reporting of symptoms and tracking of patients biomarkers, diets, physical activity and medication adherence

- Electronic reminders to take medications, eat healthy food and engage in prescribed physical activities

- Automated and clinician-activated feedback to patients and caregivers

- Auto-detection of falls and changes in patients' patterns of mobility [6].

The second available counter-measure for limiting the collateral damage from "distancing" strategies is comprehensive care management. Because it is personnel-intensive (and expensive), comprehensive care management must be rationed to a targeted subset of multi-morbid patients who are the most vulnerable to deterioration and death - and the most like to benefit from this proven service.

To target the multi-morbid patients who are most vulnerable and most likely to benefit, healthcare systems can now select from a variety of electronic risk stratification algorithms [7]. Multi-morbid patients

*Correspondence to: Chad Boult, MD, MPH, MBA, Board of Directors, International Foundation for Integrated Care, 2290 Broadview Place, Boise, ID, 83702, USA, E-mail: chad.e.boult@gmail.com

Received: October 15, 2020; Accepted: October 23, 2020; Published: October 27, 2020 
who are triaged by one of these algorithms to the highest stratum of modifiable risk are offered comprehensive care management services. Those who accept are contacted by health professionals, (e.g., nurses) by telephone, email or in-person (by teleconference, when possible) for personalized, pro-active care management, including periodic:

- Assessment of the patient's clinical, mental and safety status (with escalation to medical or other intervention, as needed)

- Assessment and facilitation of the patient's access to food, medications, equipment and needed community services

- Goal setting and care planning

- Health education and professional coaching for self-monitoring and self-management (of health and social services, medications, diet and physical activity)

- Support for family caregivers

- Coordination and integration of all of the elements of the patient's care.

Providing comprehensive care management of this intensity to the population's highest-risk stratum has been shown to improve the quality and outcomes of care [8]. During a pandemic, however, providing such care will require a temporary shift of some of the healthcare system's nursing personnel away from less urgent priorities, such as elective surgery and long-range preventive care for relatively healthy people.

Without question, nations, regions and healthcare systems need to continue their current efforts to interrupt the spread of the novel coronavirus by keeping people apart. They also have an obligation, however, to take action soon to limit the pandemic's potentially large collateral damage to people with multi-morbidity. The necessary technology and clinical processes are available, but they need to be deployed widely and strategically.
Now is the time for action! Responsible government and healthcare leaders should move rapidly to develop:

- A working knowledge of the substantial harm to our multi-morbid population that is being caused by our current anti-viral separation strategies

- Awareness of the available opportunities to deploy existing technology and comprehensive care management to limit the collateral damage of the COVID-19 war on the many members of our population with multi-morbidity.

- Pragmatic plans to implement these protective strategies as soon as possible.

\section{References}

1. Fortin M, Stewart M, Poitras M, Almirall J, Maddocks H (2012) A systematic review of prevalence studies on multimorbidity: toward a more uniform methodology. Ann Fam Med 10: 142-151. [Crossref]

2. Steinman MA, Perry L, Perissinotto CM (2020) Meeting the needs of older adults isolated at home during the COVID-19 pandemic. JAMA Intern Med 180: 819-820. [Crossref]

3. Mehrota A, Chernow M, Linetsky D, Hatch H, Cutler D (2020) The impact of the COVID-19 pandemic on outpatient visits: a rebound emerges. To the Point (blog), Commonwealth Fund.

4. Boyd CM, Wolff JL, Giovannetti E, Reider L, Weiss C, et al. (2014) Health care task difficulty among older adults with multimorbidity. Med Care 52: S118-S125. [Crossref]

5. Woolf SH, Chapman DA, Sabo RT, Weinberger DM, Hill L (2020) Excess deaths from COVID-19 and other causes, March-April 2020. JAMA 324: 510-513. [Crossref]

6. Lee JA, Choi M, Lee SA, Jiang N (2018) Effective behavioral intervention strategies using mobile health applications for chronic disease management: a systematic review. BMC Med Inform Decis Mak 18: 12

7. Brilleman SL, Gravelle H, Hollinghurst S, Purdy S, Salisbury C, et al. (2014) Keep it simple? Predicting primary health care costs with clinical morbidity measures. $J$ Health Econ 35: 109-122. [Crossref]

8. Smith SM, Wallace E, O'Dowd T, Fortin M (2016) Interventions for improving outcomes in patients with multimorbidity in primary care and community settings. Cochrane Database Syst Rev 14: CD006560. [Crossref]

Copyright: (C2020 Shadmi E. This is an open-access article distributed under the terms of the Creative Commons Attribution License, which permits unrestricted use, distribution, and reproduction in any medium, provided the original author and source are credited. 